\title{
A method for handlebars ballast calculation in order to reduce vibrations transmissibility in walk behind tractors
}

\author{
Angelo Fabbri, ${ }^{1}$ Chiara Cevoli, ${ }^{1}$ Giuseppe Cantalupo ${ }^{2}$ \\ ${ }^{1}$ Department of Agricultural and Food Science, University of Bologna, Cesena (FC); ${ }^{2}$ Fincantieri S.p.A., Trieste, Italy
}

\begin{abstract}
Walk behind tractors have some advantages over other agricultural machines, such as the cheapness and the easy to use, however the driver is exposed to high level of vibrations transmitted from handles to hand-arm system and to shoulders. The vibrations induce discomfort and early fatigue to the operator. In order to control the vibration transmissibility, a ballast mass may be added to the handles. Even if the determination of the appropriate ballast mass is a critical point in the handle design.

The aim of this research was to study the influence of the handle mass modification, on the dynamic structure behaviour. Modal frequencies and subsequent transmissibility calculated by using an analytical approach and a finite elements model, were compared. A good agreement between the results obtained by the two methods was found (average percentage difference calculated on natural frequencies equal to $5.8 \pm 3.8 \%$ ). Power tillers are made generally by small or medium-small size manufacturers that have difficulties in dealing with finite element codes or modal analysis techniques. As a consequence, the proposed analytical method could be used to find the optimal ballast mass in a simple and economic way, without experimental tests or complex finite element codes. A specific and very simple software or spreadsheet, developed on the base of the analytical method here discussed, could effectively to help the manufacturers in the handlebar design phase. The choice of the correct elastic mount, the dimensioning of the guide members and the ballast mass could be considerably simplified.
\end{abstract}

Correspondence: Chiara Cevoli, Department of Agricultural and Food Science, University of Bologna, P.zza Goidanich 60, 47521 Cesena (FC), Italy.

E-mail: chiara.cevoli3@unibo.it

Key words: Power tiller; vibrations; modal analysis; handles.

See online Appendix for additional Equations.

Received for publication: 5 September 2016.

Accepted for publication: 22 October 2016.

(C) Copyright A. Fabbri et al., 2017

Licensee PAGEPress, Italy

Journal of Agricultural Engineering 2017; XLVIII:599

doi:10.4081/jae.2017.599

This article is distributed under the terms of the Creative Commons Attribution Noncommercial License (by-nc 4.0) which permits any noncommercial use, distribution, and reproduction in any medium, provided the original author(s) and source are credited.

\section{Introduction}

Walk behind tractors (also known as walking tractor, hand tractor, pedestrian controlled or power tiller) are very common machines in small and medium size rural areas. These tractors have some advantages such as the cheapness, and the easy to use. Furthermore, the power tillers can be used in many agricultural operations like harvesting, plant protection, irrigation, threshing, and transportation (Sam and Kathirvel, 2009). In 2011 in Italy about 162,000 machines were built, 80,000 of which about agricultural tractors were built (Feder Unacoma, 2011).

Many weak points related to the use of these machines should be considered. Indeed, the operator must walk following the machine, leading to some safety threats. In Italy, excluding the injuries involving tractors, the higher percentage of the work injury in agricultural (13\%) is related to the use of power tillers (INAIL, 2013, 2014). Moreover, the operator is subjected to extreme environmental conditions, like temperature, humidity, noise and vibrations (Kanyakam and Bureerat, 2007; Dawangan and Tewari, 2010). Among these factors, the vibrations are the most important parameters to be considered. The driver of a power tiller is exposed to high levels of vibrations transmitted from handles to hand-arm system and shoulders. These vibrations induce discomfort and early fatigue to the operator. Fatigue, over a period of months or years, may cause vascular, neurological, physiological, and musculoskeletal disorders. Furthermore, it decreases efficiency and work quality (Tewari et al., 2004). The term hand-arm vibration syndrome (HAVS) is usually used for the different symptoms of this disorder (Griffin, 1996).

The hand-transmitted vibration (HTV) is distributed over a wide spectrum of frequencies and the vibration of the handles does not occur only along a preferred direction. Critical combinations of frequencies and amplitudes could cause resonance in the operator body. Low frequency vibration, lesser than $100 \mathrm{~Hz}$, are transmitted without attenuation and therefore the perception is high (Kitazaki and Griffin, 1998). With increasing of the frequency, the vibration transmissibility decreases similarly to the human sensitivity.

National and international standards give information on the relative importance of vibration frequency, magnitude, time of exposure that can influence the occurrence of HAVS. The ISO 5349 defines the guidelines to measure and evaluate human exposure, defining the details of different methods for hands and arms transmitted vibration analysis (ISO, 2001).

There are very few research works about reduction and HTV control. Most of these concern the use of isolating rubber arranged on the handles or between the engine and the machine body.

By inserting simple elastomeric rubber cushion on the handle of small tillage tool, Ragni (1994) and Xu et al. (1995) reported a vibration reduction of $35 \%$, in a single-axle tractors. Ying et al. (1998) proposed an anti-vibration device made of rubber, posi- 
tioned near the handle grip. Results showed a reduction of the frequency-weighted acceleration up to $41.1 \%$, in static conditions. Sam and Kathirvel (2009) studied the combined effect of the vibration isolators for engine, handlebars and handles, reporting a decrease in handle root mean squared vibration acceleration of about $50-60 \%$. Considering the combination of the engine and handle isolators, Tewary and Dewangan (2009) reported a vibration acceleration reduction higher than $50 \%$, in static conditions.

In order to control the HTV of the walk behind tractors, the mass, stiffness and design of the handles could be also modified (Su et al., 1989). The researchers proposed a dynamic modification approach, based on modal analysis and experimental tests. The method can reduce time of design and trial-production, it can also estimate the vibration level of the new structure.

The choose of optimal silent-block and mass distribution would require the knowledge of natural frequency of handlebars. This is a complex task to obtain experimentally. On the other hand, it could be calculated using a numerical modelling tool, but in most case the medium or small size manufacturers are not skilled at all in numerical modelling or dynamic machine analysis.

As a consequence, the aim of this research was to develop an analytical model to study the influence of the handle mass modification, on the dynamic behaviour of the structure. Especially the modal frequencies and the transmissibility related to the first vibration modes were considered. Results obtained by the modal analysis developed by using the analytical model were compared with those obtained by a finite elements model. The analytical model could be the basis for the development of a simple to use software or spreadsheet, addressed to manufacturers, useful for handlebar design and HTV reduction.

\section{Materials and methods}

Two different power tiller tractors (BRUMI $331 \mathrm{D} 14 \mathrm{~L}$ and BRUMI 327 10L; Agris Brumi s.r.1., Catania, Italy) were considered (technical data are reported in Figure 1). Adding handle mass of $0,0.73,1.09$ and $1.86 \mathrm{~kg}$ was taken into account. These values were chosen as approximately linearly distributed between 0 and the handle weight.

\section{Analytical model}

In order to reproduce the dynamical behaviour of the real structures, a simple analytical model was set up. The internal material damping was neglected, only the purely linear elastic response was considered. The transmissibility $(T r)$ was calculated according to the following usual definition:

$$
\operatorname{Tr}=\frac{1}{\left(\frac{\omega_{f}}{\omega_{n}}\right)^{2}-1}=\frac{1}{\left(\frac{f_{f}}{f_{n}}\right)^{2}-1}
$$

where $\omega$ and $f$ are pulsations and frequencies respectively. Subscripts $f$ and $n$ are related to forced actions (coming from engine) and natural behaviour (free vibrations) respectively.

The structure was schematised as in Figure 2. This mechanical system has been broken down into simpler systems, made by flexible pipes, elastically constrained, and with lumped and concentrated mass.
Natural frequencies of first, second and third vibration mode, as suggest also by Su et al. (1989), were analysed. For the first vibration mode $(I)$, the reasonable deformed shape generated by a force $F_{h}$, provided by static deformation of the system in the horizontal plane, was considered. For the second mode (II), the system deformation was provided by a force $F_{V}$ in the vertical plane. Lastly, the third mode (III) described the deformation in the vertical plane induced by a momentum:

$$
M_{t}=F_{v}(\mathrm{e}+\mathrm{d} ' \sin \gamma)
$$

The modal pulsations were calculated for the following structural subsets: i) elastic handlebars with distributed mass; ii) elastic handlebars with concentrated mass at the ends; iii) rigid handlebars with distributed mass; iv) rigid handlebars with concentrated mass at the ends.

Total modal pulsation (

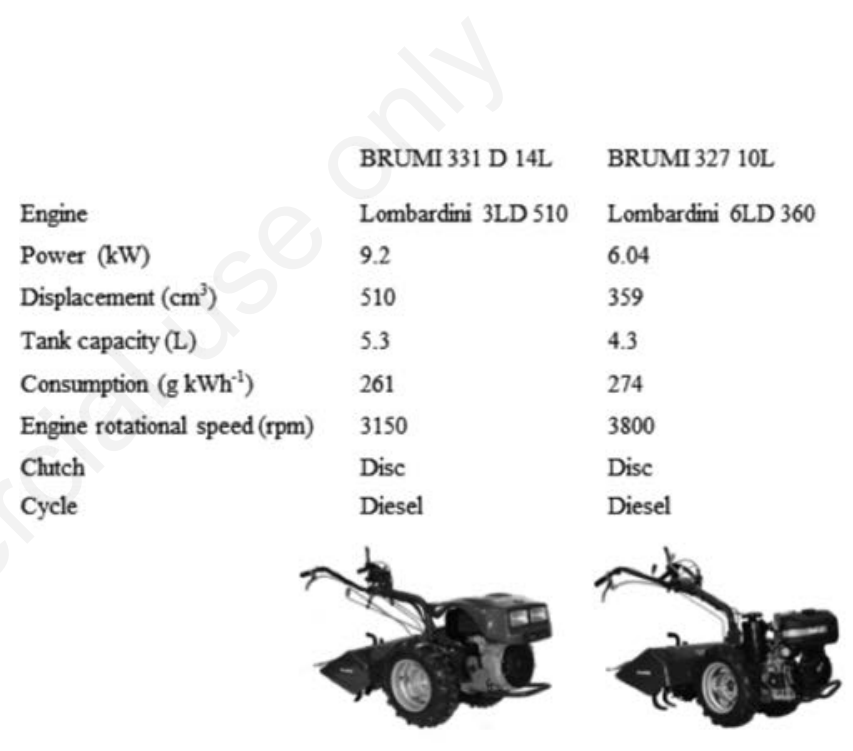

Figure 1. Main technical characteristics of the considered walk behind tractors.

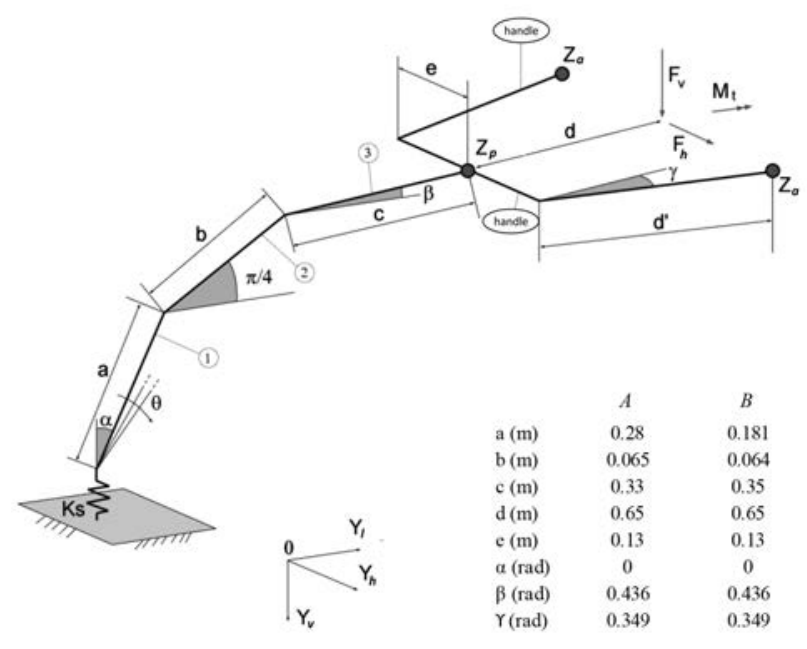

Figure 2. Handlebar geometric scheme (A: Brumi 311; B: Brumi 327). 
basis of the Dunkerley theorem (Kollar, 1999):

$\frac{1}{\omega_{T O T}^{2}}=\sum_{i} \frac{1}{\omega_{i}^{2}}$

where $\omega_{\mathrm{i}}$ is the modal pulsation for each structural subset.

The Rayleigh-Ritz method (Gould, 1995) was used for the structural subsets characterised by elastic handlebars. Pulsation values were obtained according to the energy conservation principle. For a simple oscillating system, as a cantilever beam (that is the basic element used in the model), the maximum values of kinetic $\left(T_{M A X}\right)$ and potential energy $\left(U_{M A X}\right)$ are, respectively, equal to:

$$
\begin{aligned}
& T_{\text {MAX }}=\frac{m}{2} \int_{0}^{l} \omega y(x)^{2} d x \\
& U_{\text {MAX }}=\frac{1}{2} K y_{0}^{2}
\end{aligned}
$$

For the structural subsets characterised by rigid handlebars, the Lagrange method (Lagrange, 1788) was implemented and the motion equations were expressed in generalised coordinates $(\theta)$.

\section{I vibration mode - Horizontal deformation}

Elastic handlebars with distributed masses

$$
U_{M U X}=T_{M U X} \Rightarrow \omega_{t-1}^{2}=\frac{K_{h_{\text {TOO }}} y_{h_{\text {ror }}}^{2}}{m_{\rho 1} \int_{0}^{\mathrm{b}} y_{h_{1}}^{2} d x+m_{\rho 1} \int_{0}^{c} y_{h_{2}}^{2} d s+m_{\rho 2} \int_{0}^{d^{\prime}} y_{h_{3}}^{2} d t}
$$

\section{Elastic handlebars with concentrated masses}

The concentrated masses are located in different points (Figure 2). The single ballast mass $\left(Z_{p}\right)$ placed at the end of pillar and the concentrated masses placed at the ends of the two handles $\left(Z_{a}\right)$, are separately considered.

For the single ballast mass at the pillar:

$$
U_{M A X}=T_{M A X}=>\omega_{I-2}^{2}=\frac{K_{h_{p}}}{Z_{p}}
$$

Instead, for the masses placed at the ends of the two handles:

$$
U_{M A X}=T_{M A X}=>\omega_{I-3}^{2}=\frac{K_{h_{T O T}}}{2 Z_{a}}
$$

\section{Rigid handlebars with distributed masses}

$$
\frac{d}{d t} \frac{\partial T}{\partial \dot{\theta}}-\frac{\partial T}{\partial \theta}+\frac{\partial U}{\partial \theta}=0 \Rightarrow \omega_{l-4}^{2}=\frac{K r}{\left[m_{p 1} \frac{l_{1}^{3}}{3}+\frac{2}{3} m_{p 2}\left(l_{2}^{3}-l_{1}^{3}\right)\right]}
$$

Rigid handlebars with concentrated masses

$$
\frac{d}{d t} \frac{\partial T}{\partial \dot{\theta}}-\frac{\partial T}{\partial \theta}+\frac{\partial U}{\partial \theta}=0 \Rightarrow \omega_{I-5}^{2}=\frac{K r}{\left[Z_{p} l_{1}^{2}+2 Z_{a} l_{2}^{2}\right]}
$$

Taking into account the pulsations of the four individual subset systems and applying the Dunkerly theorem, the first modal fre-

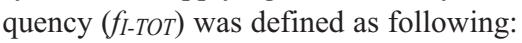

$$
f_{I-T O T}=\frac{1}{2 \pi}\left(\frac{1}{\omega_{I-1}^{2}}+\frac{1}{\omega_{I-2}^{2}}+\frac{1}{\omega_{I-3}^{2}}+\frac{1}{\omega_{I-4}^{2}}+\frac{1}{\omega_{I-5}^{2}}\right)^{-\frac{1}{2}}
$$

\section{II vibration mode - Vertical deformation}

Elastic handlebars with distributed masses

$$
U_{M X X}=T_{M X X}=>\omega_{l l-1}^{2}=\frac{K_{v_{p o r}} y_{v_{p o r}}^{2}}{m_{p 1} \int_{0}^{\mathrm{a}} y_{v_{1}}^{2} d x+m_{p 1} \int_{0}^{\delta} y_{v_{2}}^{2} d s+m_{p 1} \int_{0}^{c} y_{\nu 3}^{2} d n+2 m_{p 2} \int_{0}^{d \prime} y_{v_{1}}^{2} d t}
$$

\section{Elastic handlebars with concentrated masses}

Similarly to the first mode:

$$
\begin{gathered}
U_{M A X}=T_{M A X}=>\omega_{I I-2}^{2}=\frac{K_{v_{p}}}{Z_{p}} \\
U_{M A X}=T_{M A X} \Rightarrow \omega_{I I-3}^{2}=\frac{K_{v_{\text {TOT }}}}{2 Z_{a}}
\end{gathered}
$$

Rigid handlebars with distributed masses

$$
\begin{aligned}
& \frac{d}{d t} \frac{\partial T}{\partial \dot{\theta}}-\frac{\partial T}{\partial \theta}+\frac{\partial U}{\partial \theta}=0 \Rightarrow \\
& \omega_{l l-4}^{2}=\frac{K r}{\left\{\begin{array}{c}
m_{\rho 1} A_{26}^{2} \frac{\mathrm{a}^{3}}{3}+m_{\rho 1}\left[A_{27}^{2} \frac{\mathrm{b}^{3}}{3}+A_{28}^{2} \mathrm{~b}+2 A_{27} A_{28} \frac{\mathrm{b}^{2}}{2}\right]+ \\
m_{\rho 1}\left[A_{30}^{2} \frac{\mathrm{c}^{3}}{3}+A_{29}^{2} \mathrm{c}+2 A_{29} A_{30} \frac{\mathrm{c}^{2}}{2}\right]+2 m_{\rho 2}\left[A_{30}^{2} \frac{\mathrm{d}^{13}}{3}+A_{31}^{2} \mathrm{~d}^{1}+2 A_{30} A_{31} \frac{\mathrm{d}^{\prime 2}}{2}\right]
\end{array}\right\}}
\end{aligned}
$$

Rigid handlebars with concentrated masses

$$
\frac{d}{d t} \frac{\partial T}{\partial \dot{\theta}}-\frac{\partial T}{\partial \theta}+\frac{\partial U}{\partial \theta}=0 \Rightarrow \omega_{I I-5}^{2}=\frac{K r}{\left[Z_{p} l_{1}^{2}+2 Z_{a} l_{2}^{2}\right]}
$$

Taking into account the pulsations of the four individual systems and applying the Dunkerly theorem, the second modal fre-

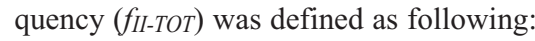


$f_{I I-T O T}=\frac{1}{2 \pi}\left(\frac{1}{\omega_{I I-1}^{2}}+\frac{1}{\omega_{I I-2}^{2}}+\frac{1}{+\omega_{I I-3}^{2}}+\frac{1}{\omega_{I I-4}^{2}}+\frac{1}{\omega_{I I-5}^{2}}\right)^{-\frac{1}{2}}$

\section{III vibration mode - Torsional deformation}

Elastic handlebars with distributed masses

$$
U_{M A X}=T_{M A X}=>\omega_{I I I-1}^{2}=\frac{\frac{1}{2} K_{t_{r o r}} y_{t_{\text {Tor }}}^{2}}{m_{p 2} \int_{0}^{\mathrm{e}} y_{t_{1}}^{2} d t+m_{p 2} \int_{0}^{\mathrm{d}} y_{t_{2}}^{2} d s}
$$

\section{Elastic handlebars with concentrated masses}

The concentrated masses are placed only at the ends of the two handles $\left(Z_{a}\right)$ :

$$
U_{M A X}=T_{M A X}=>\omega_{I I I-2}^{2}=\frac{K_{t_{\text {TrT }}}}{2 Z_{a}}
$$

Applying the Dunkerley theorem, the third modal frequency

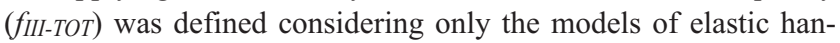
dles:

$f_{I I I-T O T}=\frac{1}{2 \pi}\left(\frac{1}{\omega_{I I I-1}^{2}}+\frac{1}{\omega_{I I I-2}^{2}}\right)^{-\frac{1}{2}}$

\section{Finite elements models}

Two simple finite element models able to reproduce the structures dynamic behaviour, were developed using SAP 90 program
(Computer and Structures Inc., University of Berkeley, CA, USA). Various mass distributions were evaluated. The handles shape was modelled by only nine Eulero-Bernoulli beam elements characterised by six degrees of freedom nodes (Figure 3). The material was considered linear elastic and consequently the natural frequencies were calculated by means of classic eigenvalues analysis (Bathe, 1996). The effect of the masses $(0,0.73,1.09$ and $1.86 \mathrm{~kg})$ arranged on the handlebars ends, was evaluated. The first five eigenfrequency, lower than $100 \mathrm{~Hz}$ were considered.

\section{Results}

The natural frequencies $(\mathrm{Hz})$ obtained by using the analytical model, as function of the ballast mass and vibration mode, are

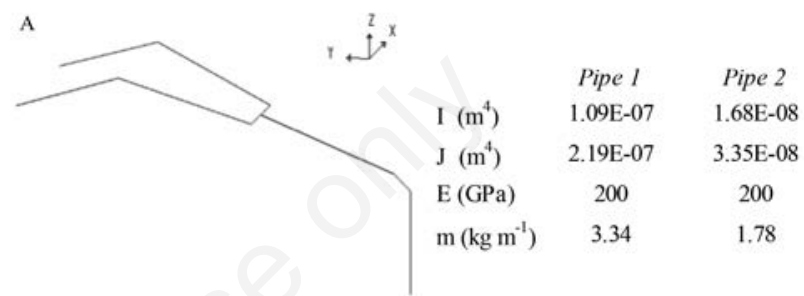

B

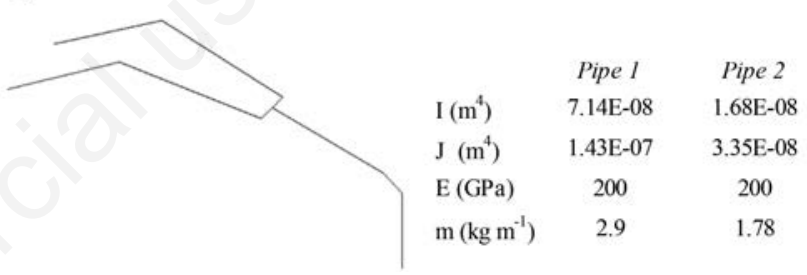

Figure 3. Scheme of handlebars simplified geometry (A: Brumi 311; B: Brumi 327) and related pipe properties (1 from the engine to the fork; 2 from the fork to the handlebars).
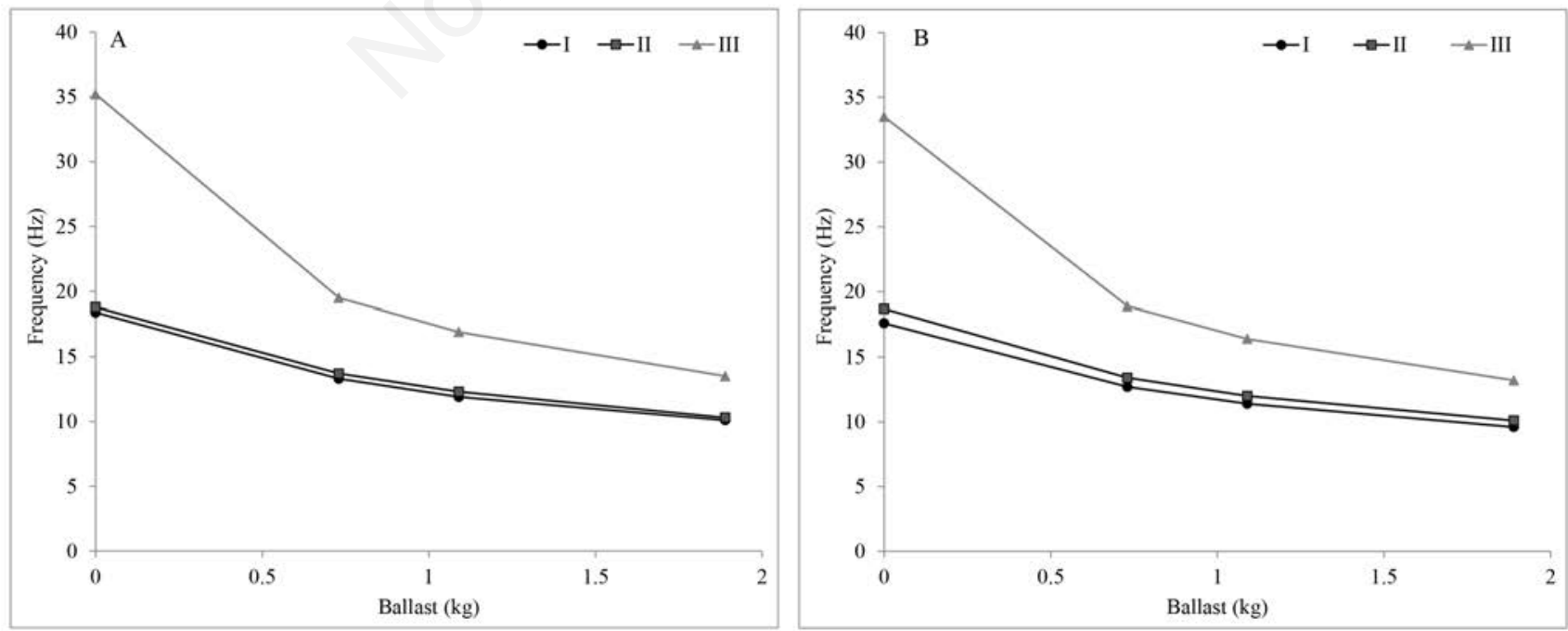

Figure 4. Natural frequencies $(\mathrm{Hz})$ calculated by analytical method, as function of ballast mass and vibration mode (A: Brumi 311; B: Brumi 327). 
reported in Figure 4. For both walk behind tractors, the natural frequencies decrease with the increase of the ballast mass. Considering a ballast of $1.86 \mathrm{~kg}$ an average reduction of $51 \%$, was observed for all vibration modes. The natural frequency decreases of about $45 \%$ due to a ballast mass of only $0.73 \mathrm{~kg}$ (third vibration mode, torsional deformation).

Concerning the finite element models, the first five vibration modes were considered and the results are shown in Figure 5. Here too, the natural frequency values decrease with the increase of the ballast mass, up to $58 \%$ (ballast of $1.86 \mathrm{~kg}$, fourth vibration mode). The reduction is accentuated with the increase of the vibration mode (except that for the fifth vibration mode). With the same ballast mass, similar natural frequencies were obtained for the first two vibration modes and for the third and fourth vibration modes (maximum difference of 7\%). As you would expect, the natural frequency increase with the vibration order, up to $77 \mathrm{~Hz}$ (fifth modes, no ballast).

In order to compare the results, the absolute percentage differences between natural frequencies calculated by using finite elements model and the analytical approach, were evaluated and reported in Table 1. The average percentage difference is limited and equal to $5.8 \pm 3.8 \%$ (standard deviation); maximum and minimum are $13.8 \%$ and $1.4 \%$, respectively. Monotonic trends were not observed as function of ballast mass and vibration mode.

Transmissibility $(\mathrm{Tr})$ obtained for each vibration mode, frequency of forced excitations from the engine (first and second order), and ballast mass, were evaluated and reported in Tables 2 (analytical method) and 3 (finite element model). Frequencies of forced excitations from the engine of $52.5 \mathrm{~Hz}$ (M1) and $105.0 \mathrm{~Hz}$

Table 1. Percentage differences between natural frequencies obtained with analytical method and finite element model.

\begin{tabular}{lccccc} 
Power tiller model & Vibration mode & \multicolumn{5}{c}{ Ballast (kg) } \\
& & 0 & 0.73 & 1.09 & 1.89 \\
\multirow{3}{*}{ Brumi 311 } & I & 5.12 & 0.62 & 1.46 & 3.96 \\
& II & 9.74 & 2.89 & 1.69 & 0.57 \\
\multirow{2}{*}{ Brumi 327 } & III & 4.56 & 6.76 & 7.67 & 9.62 \\
& $I$ & 13.13 & 5.75 & 4.29 & 2.29 \\
& II & 13.77 & 6.53 & 5.26 & 3.31 \\
\hline
\end{tabular}

Table 2. Transmissibility calculated by using the data obtained by the analytical method.

\begin{tabular}{|c|c|c|c|c|c|c|}
\hline \multirow[t]{2}{*}{ Power tiller model } & \multirow[t]{2}{*}{ Vibration mode } & \multirow[t]{2}{*}{ Frequency of forced excitations from the engine } & \multicolumn{4}{|c|}{ Ballast (kg) } \\
\hline & & & 0 & 0.73 & 1.09 & 1.89 \\
\hline \multirow[t]{6}{*}{ Brumi 311} & I & M1 & 0.14 & 0.07 & 0.05 & 0.04 \\
\hline & & M2 & 0.03 & 0.02 & 0.01 & 0.01 \\
\hline & II & M1 & 0.15 & 0.07 & 0.06 & 0.04 \\
\hline & & M2 & 0.03 & 0.02 & 0.01 & 0.01 \\
\hline & III & M1 & 0.82 & 0.16 & 0.12 & 0.07 \\
\hline & & M2 & 0.13 & 0.04 & 0.03 & 0.02 \\
\hline \multirow[t]{6}{*}{ Brumi 327} & $I$ & $\mathrm{~N} 1$ & 0.08 & 0.04 & 0.03 & 0.02 \\
\hline & & N2 & 0.02 & 0.01 & 0.01 & 0.01 \\
\hline & II & N1 & 0.10 & 0.05 & 0.04 & 0.03 \\
\hline & & N2 & 0.02 & 0.01 & 0.01 & 0.01 \\
\hline & III & N1 & 0.39 & 0.10 & 0.07 & 0.05 \\
\hline & & $\mathrm{N} 2$ & 0.08 & 0.02 & 0.02 & 0.01 \\
\hline
\end{tabular}

$\mathrm{M} 1,52.5 \mathrm{~Hz} ; \mathrm{M} 2,105.0 \mathrm{~Hz} ; \mathrm{N} 1,63.3 \mathrm{~Hz} ; \mathrm{N} 2,126.7 \mathrm{~Hz}$.

Table 3. Transmissibility calculated by using the data obtained by the finite element model.

\begin{tabular}{|c|c|c|c|c|c|c|}
\hline Power tiller model & Vibration mode & Frequency of forced excitations from the engine & & Ballas & & \\
\hline & & & 0 & 0.73 & 1.09 & 1.89 \\
\hline Brumi 311 & $I$ & M1 & 0.16 & 0.07 & 0.05 & 0.04 \\
\hline & & M2 & 0.04 & 0.02 & 0.01 & 0.01 \\
\hline & II & M1 & 0.19 & 0.08 & 0.06 & 0.04 \\
\hline & & M2 & 0.04 & 0.02 & 0.01 & 0.01 \\
\hline & III & M1 & 0.70 & 0.19 & 0.14 & 0.09 \\
\hline & & M2 & 0.11 & 0.04 & 0.03 & 0.02 \\
\hline & $I V$ & M1 & 1.40 & 0.26 & 0.18 & 0.11 \\
\hline & & M2 & 0.17 & 0.05 & 0.04 & 0.03 \\
\hline & V & M1 & 2.55 & 79.37 & 8.14 & 2.57 \\
\hline & & M2 & 0.70 & 0.34 & 0.29 & 0.22 \\
\hline Brumi 327 & I & $\mathrm{N} 1$ & 0.11 & 0.05 & 0.04 & 0.02 \\
\hline & & N2 & 0.03 & 0.01 & 0.01 & 0.01 \\
\hline & II & $\mathrm{N} 1$ & 0.13 & 0.05 & 0.04 & 0.03 \\
\hline & & N2 & 0.03 & 0.01 & 0.01 & 0.01 \\
\hline & III & $\mathrm{N} 1$ & 0.37 & 0.12 & 0.09 & 0.06 \\
\hline & & N2 & 0.07 & 0.03 & 0.02 & 0.01 \\
\hline & IV & $\mathrm{N1}$ & 0.67 & 0.16 & 0.12 & 0.07 \\
\hline & & N2 & 0.11 & 0.04 & 0.03 & 0.02 \\
\hline & V & $\mathrm{N} 1$ & 3.22 & 13.05 & 4.33 & 1.85 \\
\hline & & N2 & 0.57 & 0.30 & 0.25 & 0.19 \\
\hline
\end{tabular}

$\mathrm{M1}, 52.5 \mathrm{~Hz} ; \mathrm{M} 2,105.0 \mathrm{~Hz} ; \mathrm{N1}, 63.3 \mathrm{~Hz} ; \mathrm{N} 2,126.7 \mathrm{~Hz}$. 

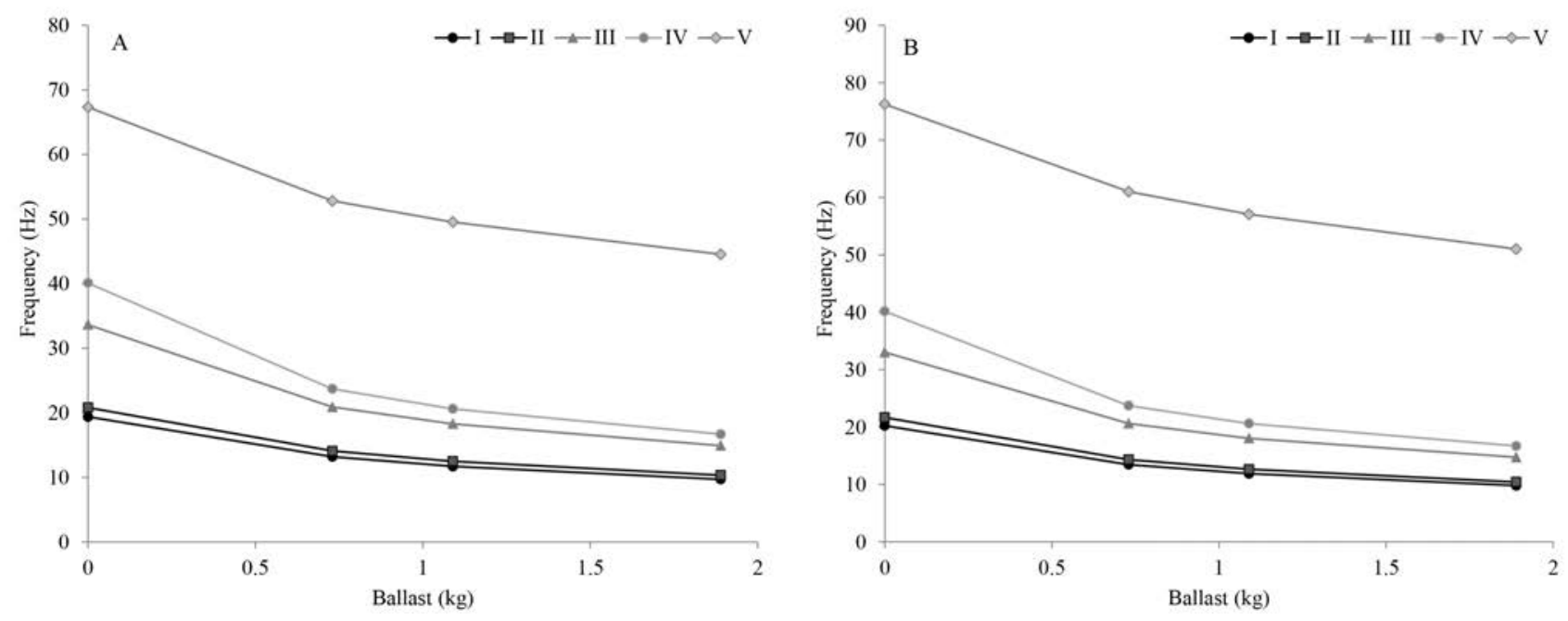

Figure 5. Natural frequencies $(\mathrm{Hz})$ calculated by finite element model, as function of ballast mass and vibration mode (A: Brumi 311 ; B: Brumi 327).

(M2), and of $63.3 \mathrm{~Hz}(\mathrm{~N} 1)$ and $126.7 \mathrm{~Hz}$ (N2) were considered, for the Brumi 311 (engine rotation speed of $3150 \mathrm{rpm}$ ) and the Brumi 217 (engine rotation speed of $3800 \mathrm{rpm}$ ), respectively. The transmissibility value depends also on the engine rotational speed, but it has to be considered that power tillers are almost never used at partial loadings.

Considering the first three vibration modes, the transmissibility values are lower than 1 (insulation value) and they asymptotically decrease with the increase of the ballast mass. Average reductions of $77.1 \pm 8.2 \%$ and $78.6 \pm 5.3 \%$, were achieved (ballast mass of $1.89 \mathrm{~kg}$ ), for the analytical approach and finite element model, respectively. However, high reductions were reached also with a ballast mass of $0.73 \mathrm{~kg}$ (up to $68.1 \pm 12.4 \%$ ). In general, very similar results were obtained for both walk behind tractors.

By using the finite element models, also the transmissibility obtained for the fourth and fifth vibration modes were calculated. For the fourth mode, similar trend (transmissibility that decreases exponentially with the increase of ballast mass) and values (lower than 1) obtained for the first three vibration modes, were observed, except for the value calculated for the Brumi 311 (M1) without ballast. In this latter case, the ratio between frequency of forced excitations from the engine $(52.5 \mathrm{~Hz})$ and natural frequency $(40.1$ $\mathrm{Hz}$ ) was lower than 1.4 and the transmissibility was systematically higher than 1 (amplification condition). The values achieved for the fifth vibration modes and the first frequency of forced excitations from the engine $(52.5$ and $63.3 \mathrm{~Hz}$, for Brumi 311 and Brumi 327, respectively), are higher than 1 and they do not decrease exponentially as function of the ballast mass. Particularly, for the ballast mass of $0.73 \mathrm{~kg}$, highest transmissibility values were observed (79.37 and 13.05, for Brumi 311 and Brumi 327, respectively). In these cases the ratios between frequency of forced excitations from the engine and natural frequency are near to $1(0.99$ and 1.04) that represents the resonance condition.

\section{Conclusions}

It is possible to reduce the power tiller vibration transmissibil- ity by using ballast masses arranged on the handles. This could be useful for manufacturing companies in order to improve the safety in a simple and economic way. However the identification of the appropriate ballast mass is a critical point in the design phase, being essential to avoid the masses that modifies natural frequency of handles causing conditions close to resonance. The determination of natural handles frequencies requires a complex measurement procedure of modal analysis, or the development of a calculation model. Within the present research, numerical and analytical models were developed to evaluate the first natural frequencies of a generic handle parametrically described. The agreement between the results obtained by the two models, in terms of average percentage difference, was $5.8 \pm 3.8 \%$.

For a small or medium-sized manufacturer it could be a very hard task to measure the natural frequencies or to develop an own calculation mode, both numerical and analytical.

As a consequence a very simple calculation spreadsheet or software, developed on the base of the analytical method here described, could effectively to help the manufacturer during the design phase as regard the choice of the correct elastic mounting, the dimensioning of the driving members, and the ballast masses.

\section{Nomenclature}

$A$ equation parameter

$B$ equation parameter

$E$ elasticity modulus (Pa)

$F$ force (N)

$G$ shear modulus (Pa)

$K$ stiffness $\left(\mathrm{N} \mathrm{m}^{-1}\right)$

$K r$ torsional stiffness $(\mathrm{N} \mathrm{m})$

$I$ moment of inertia $\left(\mathrm{m}^{4}\right)$

$L$ pipe length (m)

$M$ momentum ( $\mathrm{N} \mathrm{m}$ )

$T$ kinetic energy (J)

$U$ potential energy $(\mathrm{J})$

$Z$ ballast mass (kg)

$\operatorname{Tr}$ transmissibility

\begin{tabular}{ll}
\multicolumn{2}{c}{ Subscript } \\
$a$ & handle \\
$f$ & driving \\
$n$ & natural \\
$p$ & pillar \\
$s$ & silent block \\
$h$ & horizontal \\
$t$ & torsional \\
$v$ & vertical \\
0 & extremity \\
$I$ & first order \\
II & second order \\
III & third order \\
TOT & total
\end{tabular}


$f \quad$ frequency $(\mathrm{Hz})$

$l$ length (m)

$m$ mass per unit length $\left(\mathrm{kg} \mathrm{m}^{-1}\right)$

$y$ displacement $(\mathrm{m})$

$\omega$ pulsation $\left(\mathrm{s}^{-1}\right)$

\section{References}

Bathe K.J. 1996. Finite element procedures. Prentice Hall, Inc., Upper Saddle River, New Jersey, NJ, USA.

Dewangan K.N., Tewari V.K. 2010. Handle grips for reducing hand-transmitted vibration in hand tractor. IAEJ. 19:48-57.

Feder Unacoma 2011. Macchine per l'agricoltura e per il movimento terra, andamento dei settori industriali. General Meeting. Feder Unacoma, Bologna, Italy.

Gould S.H. 1995. Variational methods for eigenvalue problems: an introduction to the methods of Rayleigh, Ritz, Weinstein, and Aronszajn. Dover Publications, Inc., Mineola, NY, USA.

Griffin M.J. 1996. Hand book of human vibration. Academic Press, London, UK.

INAIL (Istituto nazionale Assicurazione Infortuni sul Lavoro). 2013. Report annuale sugli infortuni mortali e con feriti gravi verificatisi nel 2013 nel settore agricolo e forestale. Dipartimento Innovazioni Tecnologiche e Sicurezza degli Impianti, Prodotti e Insediamenti Antropici dell'INAIL. INAIL, Roma, Italy.

INAIL (Istituto nazionale Assicurazione Infortuni sul Lavoro). 2014. Report annuale sugli infortuni mortali e con feriti gravi verificatisi nel 2014 nel settore agricolo e forestale. Dipartimento Innovazioni Tecnologiche e Sicurezza degli Impianti, Prodotti e Insediamenti Antropici dell'INAIL. INAIL, Roma, Italy.

ISO 5349 2001. Mechanical vibration-measurement and evalua- tion of human exposure to hand transmitted vibration, part 1: general requirements. International Standardisation Organisation (ISO) Geneva, Switzerland.

Kanyakam S., Bureerat S. 2007. Passive vibration suppression of a walking tractor handlebar structure using multiobjective PBIL. pp 4162-4169 in Proc. IEEE Congress on Evolutionary Computation, Suntec, Singapore.

Kitazaki S., Griffin M.J. 1998. Resonance behaviour of the seated human body and effects of posture. J. Biomech. 31:143-9.

Kollar L. 1999. Structural stability in engineering practice. CRC Press, Boca Raton, FL, USA.

Lagrange J.L. 1788. Mécanique analytique. Chez La Veuve Desaint, Paris, France.

Ragni L. 1994. Vibrazioni trasmesse al sistema mano-braccio da motocoltivatori. Parte quarta: un intervento di bonifica. Riv. di Ing. Agr. 1:18-26.

Sam B., Kathirvel K. 2009. Development and evaluation of vibration isolators for reducing hand transmitted vibration of walking and riding type power tillers. Biosyst. Eng. 103:427-37.

Su Q., Zhang Y., Xu Q., Zhang Z. 1989. Dynamic modification applied to the design of the handle of a walking tractor. Trans. Chinese Soc. Agric. Machine. 20:9-14.

Tewari V.K., Dewangan K.N. 2009. Effect of vibration isolators in reduction of work stress during field operation of hand tractor. Biosyst. Eng. 103:146-58.

Tewari V.K., Dewangan K.N., Karmakar S. 2004. Operator's fatigue in field operation of hand tractors. Biosyst. Eng. 89:1-11.

Xu F., Zhang L., Yibin Y, Ragni L., Vassalini G. 1995. Anti-vibration solutions for ride-on walking tractor. In Proc. Int. Conf. on Structural Dynamics, Vibration, Noise and Control, Hong Kong.

Ying Y., Zhang L., Xu F., Dong M. 1998. Vibration characteristics and hand transmitted vibration reduction of walking tractor. Trans. ASAE. 41:917-22. 